\title{
Study on the Denoising Processing of Ultrasonic Testing Signal for Standing Trees Based on Wavelet Transform
}

\author{
Wenshu Lin, Haokai Xu and Jinzhuo Wu* \\ College of Engineering and Technology, Northeast Forestry University, Harbin \\ 150040, China \\ linwenshu@126.com,xhk1512@163.com \\ *Corresponding author: wujinzhuo1980@163.com
}

\begin{abstract}
To remove the impact of noise on the ultrasonic testing signals of standing trees, wavelet transform method was used to eliminate the noise in the collected ultrasonic signals in the field. In order to achieve the best denoising effect, four kinds of wavelet base denoising parameters including Daubechies (db), Symlets (sym), Coiflets (coif), and Discrete Meyer (dmey) were compared, and the best denoising effect was obtained with $d b 3$ wavelet base. The variations of denoising parameters corresponding to the number of db3 wavelet decomposition levels (1-8) were further analyzed and the decomposition level 4 was demonstrated the best. Meanwhile, the effects of wavelet denoising under different threshold states were compared and the hard rigrsure threshold was demonstrated the best. Experimental results showed that the wavelet transform can effectively remove noise hidden in the ultrasonic signal and improve the denoising effect by selecting reasonable parameters, which laid some initial groundwork for efficient extraction of useful information from the ultrasonic signals.
\end{abstract}

Keywords: Ultrasonic, Standing tree, Wavelet transform, Denoising

\section{Introduction}

Wood defects can affect the quality of wood materials to different extent, making them reduce or even completely lose their value. In order to save the limited timber resources and improve the utilization of wood, for decades, people have been working on some quick and accurate methods to detect wood defects. Therefore, the study of detecting internal defects in the wood has very important practical significance. Traditional wood defects detection is conducted mostly by using artificial methods, but these methods are time consuming, which require harsh testing conditions, and have poor stability and low accuracy. Some tests even have to be conducted by destroying the wood samples being tested, so these detection methods cannot meet the needs of non-destructive, rapid and continuous detection during timber production. Wood non-destructive testing (WNDT) technique was gradually emerged in the 1960s, which was used for detecting the growth characteristics, physical properties, mechanical properties, and defects of wood and wood components [1]. With the continuous development of electronics, optics, and computer technology, currently more than dozens of WNDT technologies have been applied in testing wood properties, such as ray inspection, ultrasonic testing, magnetic resonance detection, microwave detection, stress wave detection, and acoustic emission testing [2]. Based on the related literature review at home and abroad, it is concluded that ultrasonic detection method is one of the most widely used methods in WNDT techniques. It can be applied in detecting the surface and internal decay of wood, knots, resin, bark, holes, and other defects in the wood [3-6]. Although the application of ultrasonic in wood defects

* Corresponding Author 
detection is very extensive, the study on the defect detecting for the healthy standing trees or trees with internal defects is still very limited.

While propagating in the timber, large amount of information including the relevant timber properties, internal structure, and composition will be recorded by the ultrasonic wave. Accurate determination on the changes of the acoustic parameters can help infer the performance, internal structure, and composition of wood. However, useful information is often hidden in the acquired signal, since the sensor received signal is always accompanied by random noise. Of course, the most effective and thorough approach is to eliminate the possibility of producing noise. But due to the impacts of the detection instrument itself, testing conditions in the field, ambient noise, and other factors, it is often difficult to be eradicated. Therefore, only the signal processing techniques can be used to remove or reduce the noise in the detection signal and greatly improve the reliability of detection. Currently, the Fourier transform is widely used in signal processing, especially for relatively stable periodic signal. However, it cannot reflect the mutation characteristics of the signal, or describe the local features in the time domain, thus it is difficult to meet the time-domain and local variation characteristics in the frequency domain. At present, some researchers began to use wavelet analysis for ultrasonic signals processing, especially in signal denoising. Wavelet transform method has unique advantages in signal denoising, which has good time-frequency localization properties and can be focused in any detail signal.

Among the wavelet transform denoising methods, the wavelet threshold denoising is simple, requiring less calculation, and has extensive adaptation, which is one of the most widely used wavelet denoising methods. Therefore, the purpose of this paper is to conduct wavelet threshold denoising on the ultrasonic testing signal collected in the field by using the wavelet transform, and to measure the effect of denoising by signal-to-noise ratio (SNR) and other indicators, and to determine the optimal wavelet base, the number of wavelet decomposition levels and the type of threshold.

\section{Material and Method}

\subsection{Ultrasonic Signal Acquisition and Data Preprocessing}

The ultrasonic instrument used in this study was RSM-SY5 ultrasonic detector produced by Wuhan Institute of Rock Mechanics in China, as shown in Figure 1 (a). In order to effectively detect the standing trees, the probes of the RSM-SY5 ultrasonic detector were modified, as shown in Figure 1 (b). The original probe of the ultrasonic longitudinal wave is planar. However, the barks of logs and standing trees will result in a large gap between the ultrasonic probe and the object to be measured, which has a significant negative impact on the detection accuracy. Thus, a steel conical waveguide rod was connected to the original flat probe. The contact surface of the cone cusp is very small, so the ultrasonic longitudinal wave from the cone tip to the standing tree can be taken as a point source.

The standing trees were selected from the experimental forest farm at Northeast Forestry University, China $\left(126^{\circ} 62^{\prime} \sim 126^{\circ} 63^{\prime} \mathrm{E}, 45^{\circ} 711^{\prime} \sim 45^{\circ} 72^{\prime} \mathrm{N}\right.$, with an average elevation of $142 \mathrm{~m}$ ). A total of forty standing trees with diameter of $0.40-0.55 \mathrm{~m}$ were selected and ultrasonic testing was conducted at tree height of $1.30 \mathrm{~m}$ in order to determine the location of the internal defects. Before testing, some testing parameters were set up for the ultrasonic detector, such as trigger mode (inside trigger), gain value (1000), high-pass filter frequency $(10 \mathrm{kHz})$, low-pass filter frequency $(10 \mathrm{kHz})$, signal sampling point (2048), and sampling period (1us).

The ultrasonic signal acquisition results were saved in RWV format and the data structure was a $16 \times 128$ matrix. In order to facilitate the later data processing, the data structure was transformed into a $1 \times 2048$ matrix in MATLAB software. The transform functions are: $a=a^{\prime}, Y=$ reshape $(a, 1,2048)$, where $a$ is the original signal, and $Y$ is 
transformed array. Once data preprocessing on the collected signal is done, wavelet denoising can be started.

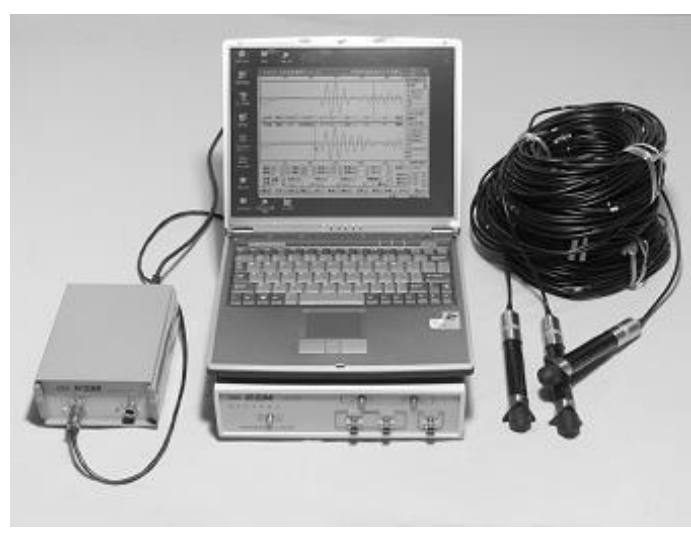

(a) Ultrasonic testing detector

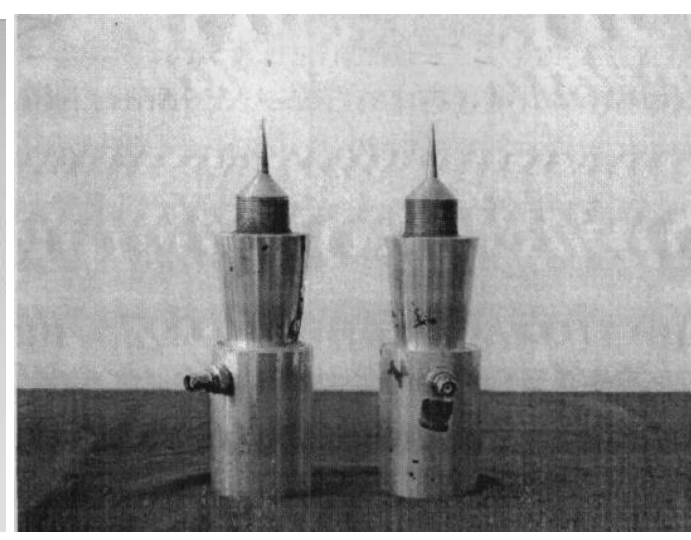

(b) The modified ultrasound probe

Figure 1. Ultrasonic Testing System

\subsection{The Principles of Wavelet Denoising}

Wavelet transform is developed from the Fourier transform, which is a new method of signal analysis. The basic idea is to decompose the original signal into a series of primitive signals with good positioning of frequency domain and to use various features of the primitive signals to characterize the local features of the original signal, and to realize localization analysis on the time-frequency domain of the signal [7-8]. The wavelet transform is to expand the arbitrary function $f(t) \in L^{2}(R)$ on the wavelet base $\psi(t)$. The mathematical expression for the wavelet transform is defined as:

$W f(\alpha, \beta)=\left\{f(t), \psi_{\alpha, \beta}(t)\right\}$

This expansion is called continuous wavelet transform for the $f(t)$ function. However, in the practical applications, especially in the computer realization, continuous wavelet must be made to be discrete. The mathematical expression for the discrete wavelet transform is given by:

$\left(W_{\psi} f\right)(\alpha, \beta)=\left\{f, \psi_{\alpha, \beta}\right\}=\left|\alpha_{0}\right|^{-\gamma / 2} \int_{-\infty}^{+\infty} f(t) \overline{\psi\left(\alpha_{0}^{-\gamma} t-\tau \beta_{0}\right)} d t$

After decomposing the signal into different levels, the wavelet coefficients $\left(W_{\psi} f\right)(\alpha, \beta)$ can be obtained, then inverse transform is conducted on the coefficients and the signal reconstruction is completed. The mathematical expression is:

$f(t)=\sum_{\gamma, \tau}\left(w_{\psi} f\right)(\alpha, \beta) \psi_{\gamma, \tau}(t)$

Where, $L^{2}(R)$ represents the square-integrable real-value space, that is the energy limited signal space, $\alpha$ and $\beta$ are the stretch factor and the translation factor for $\psi(t)$, respectively. $\alpha, \beta$ define the width of wavelets and center position, respectively, where $\alpha=\alpha_{0}^{\gamma}, \beta=\tau \beta_{0} \alpha_{0}^{\gamma}$.

In the denoising process using wavelet transform, the selection and quantification of the threshold can determine the final quality of the signal to some extent. To determine 
the optimal denoising method, three types of adaptive thresholds were adopted to denoise and reconstruct the ultrasonic signal. Each threshold is calculated as follows:

Sqtwolog threshold

$\lambda_{1}=\delta \sqrt{2 \ln n}$

where, $\delta$ is noise intensity, $n_{\text {is }}$ the number of wavelet coefficients.

Rigrsure threshold

It is assumed that $W=\left[W_{1}+W_{2}, \cdots, W_{n}\right]$ and $W_{1} \leq W_{2} \leq \cdots W_{n}$, and the elements in $W$ are the squared wavelet coefficients. $\mathrm{R}$ is defined as the risk vector, $r_{i}=\frac{\left[n-2 i-(n-i) W_{i}+\sum_{m=1}^{i} W_{m}\right]}{n}$

so the threshold can be calculated by:

$\lambda_{2}=\delta \sqrt{\omega_{a}}$

Minimaxi threshold

$\lambda_{3}=\left\{\begin{array}{lc}0 & n<32 \\ \delta\left(0.396+0.1829 \log 2^{n}\right) & n \geq 32\end{array}\right.$

\subsection{Evaluation of Denoising}

In the process of noise removal, some effective information may be regarded as ultrasonic noise and eliminated, so it is necessary to evaluate the effect of denoising. In this paper, the signal-to-noise ratio (SNR) and root mean squared error (RMSE) were used as computing standards to evaluate the effect of wavelet denoising. The larger the SNR value and the smaller the RMSE value, the better the noise removal and effective information of the signal retained is more complete, which means that the denoising effect of this method is better. The expressions for SNR and RMSE are defined as:

$$
S N R=10 * \log \left(\frac{\sum_{i=1}^{n} \hat{f}^{2}(i)}{\sum_{i=1}^{n}\left(f^{2}(i)-\hat{f}(i)\right)^{2}}\right)
$$

$R M S E=\sqrt{\frac{1}{n} \sum_{i=1}^{n}\left((f(i)-\hat{f}(i))^{2}\right.}$

where, $f(i)$ is the collected ultrasonic signal, and $\hat{f}(i)$ is the ultrasonic signal after denoising.

\section{Results and Analysis}

\subsection{Effects of Denoising by Different Wavelet Bases}

The appropriateness of wavelet base selection is directly related to the effect of denoising. In this paper, four commonly used wavelet bases were selected to decompose the ultrasonic signal into two levels and process denoising, including Daubechies (db), 
Symlets (sym), Coiflets (coif), and Discrete Meyer (dmey). Here, Daubechies is db3, Symlets is sym3, and Coiflets is coif3. The effects of denoising by the four wavelet bases are presented in Table 1 . The order of SNR value was dmey<coif $3<\operatorname{sym} 3<\mathrm{db} 3$, and the maximum value was 34.9167. While, the order of RMSE value was $\mathrm{db} 3<\operatorname{sym} 3<$ coif3<dmey, and the minimum value was 3.2195. The results showed that among the four wavelet bases, the denoising effect with $\mathrm{db} 3$ wavelet base was the best.

Table 1. The Effects of Denoising by Different Wavelet Bases

\begin{tabular}{ccccc}
\hline Parameters & $\mathrm{db} 3$ & sym3 & coif3 & dmey \\
\hline SNR & 34.9167 & 34.7283 & 34.7032 & 34.4814 \\
RMSE & 3.2195 & 3.291 & 3.3006 & 3.3859 \\
\hline
\end{tabular}

\subsection{Effects of Denoising by Different Decomposition Levels}

In the denoising process by wavelet transform, ultrasonic portable noise may not be completely removed with fewer decomposition levels, while a part of effective information may be rejected as noise with excessive decomposition levels. Therefore, the determination of the wavelet decomposition levels is critical to the effect of ultrasonic denoising. In this paper, the db3 wavelet in the toolbox of Matlab software was applied to conduct fixed soft and hard threshold denoising under different decomposition levels in order to find the best decomposition levels.

Table 2. The Effects of Denoising by Different Decomposition Levels

\begin{tabular}{lllllllll}
\hline Parameters & 1 & 2 & 3 & 4 & 5 & 6 & 7 & 8 \\
\hline SNR & 34.9167 & 35.2398 & 35.9961 & 37.5451 & 34.7662 & 34.6563 & 34.63 & 34.6072 \\
RMSE & 3.2195 & 3.1033 & 2.8477 & 2.3886 & 3.2749 & 3.3161 & 3.3259 & 3.3343 \\
\hline
\end{tabular}

As shown in Table 2, SNR and RMSE values were different from each other decomposition level, which means that the effects of denoising of db3 wavelet under 1-8 wavelet decomposition levels were different. With the increase of decomposition levels, SNR showed an increasing trend, while RMSE showed a decreasing trend. When the number of decomposition levels was equal to four, SNR reached the maximum value of 37.5451 , and RMSE reached the minimum value of 2.3886. However, when the number of decomposition levels was greater than four, SNR showed a decreasing trend and RMSE showed an increasing trend with the increase of the decomposition levels. The reason for this phenomenon was that the denoising effect of db3 wavelet enhanced with the increase of decomposition levels and achieved the best when the decomposition levels reached four in this study. However; when the number of decomposition levels surpassed four, with the increase of decomposition levels, a part of the effective information was rejected as noise and the effective information being removed was also increasing, resulting in the decrease of the effect of wavelet denoising.

\subsection{Effects of Denoising by Different Thresholds}

After determining the wavelet base function and wavelet decomposition levels, the selection and quantification of threshold play a decisive role in the wavelet denoising effect. In this paper, under the db3 wavelet and four decomposition levels, three types of adaptive thresholds including sqtwolog threshold, rigrsure threshold, and minimaxi threshold were used in combination with soft and hard quantization thresholds to denoise the signal to determine the best method of denoising. The related denoising parameters are presented in Table 3 . 
Table 3. The Effects of Denoising by Different Thresholds

\begin{tabular}{ccccccccc}
\hline \multirow{2}{*}{$\begin{array}{c}\text { Denoising } \\
\text { methods }\end{array}$} & \multicolumn{2}{c}{$\begin{array}{c}\text { sqtwolog } \\
\text { threshold }\end{array}$} & & \multicolumn{2}{c}{ rigrsure threshold } & & \multicolumn{2}{c}{$\begin{array}{c}\text { minimaxi } \\
\text { threshold }\end{array}$} \\
\cline { 2 - 3 } & SNR & RMSE & & SNR & RMSE & & SNR & RMSE \\
\hline $\begin{array}{c}\text { Soft } \\
\text { threshold }\end{array}$ & 37.5451 & 2.3886 & & 54.514 & 0.3417 & & 39.1916 & 1.9795 \\
$\begin{array}{c}\text { Hard } \\
\text { threshold }\end{array}$ & 61.831 & 0.1474 & & 89.9371 & 0.0058 & & 67.3438 & 0.0781 \\
\hline
\end{tabular}

It was shown from Table 3 that regardless of the type of threshold selected, the SNR value after denoising for the quantized wavelet by a hard threshold was larger than that by a softer threshold, while root mean square error vice versa, which indicated that the quantization effect for the hard threshold was better than soft threshold. After quantifying by the hard threshold, the order of the SNR obtained after denoising is sqtwolog threshold < minimaxi threshold < rigrsure threshold, and the order of the RMSE is rigrsure threshold < minimaxi threshold < sqtwolog threshold, and soft threshold is also the same, which showed that among the three types of adaptive thresholds the effect of denoising by rigrsure threshold was the best. To sum up, the best way for denoising was the rigrsure hard threshold, and the SNR and RMSE were 87.9371 and 0.0058, respectively.

\subsection{Effect of Wavelet Transform Denoising}

In order to confirm the effect of wavelet transform denoising, the signals before and after the rigrsure denoising were reconstructed, as shown in Figure 2. Figure 2(a) and Figure 2(b) show the signals before and after the rigrsure with hard quantization threshold denoising, respectively. Although part of the denoising effect can be seen, the important information such as specific point or location cannot be identified from the signals In order to identify the hidden defects and the presence of noise more effectively in the ultrasonic signal, low-frequency approximation signal and high-frequency signal were plotted. Figure 2(c) is ultrasonic frequency coefficient with four decomposition levels. When x-axis was between 950-1200, the concave of ultrasonic was very significant, which was caused by the attenuation of some ingredients in the specified frequency band signal in the defective portion and indicated the presence of internal defects in the standing tree. Figure 2(d) is the high-frequency detail signal which shows mostly noise in the signal.

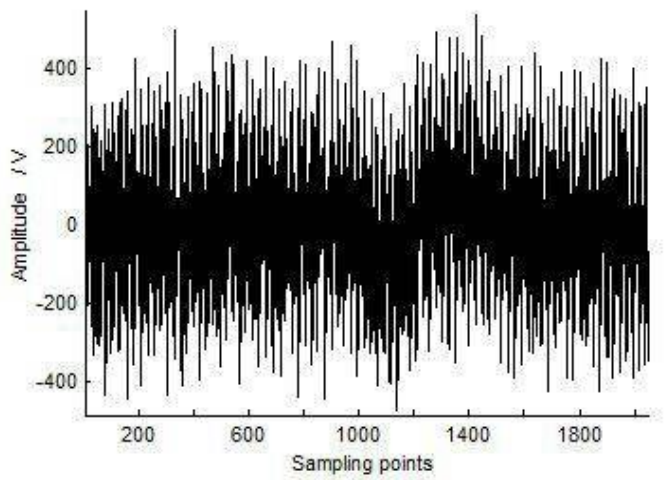

(a) Original signal

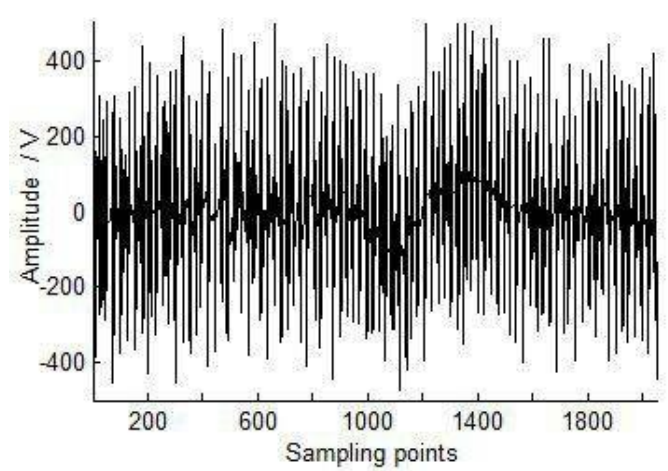

(b) After denoising signal 


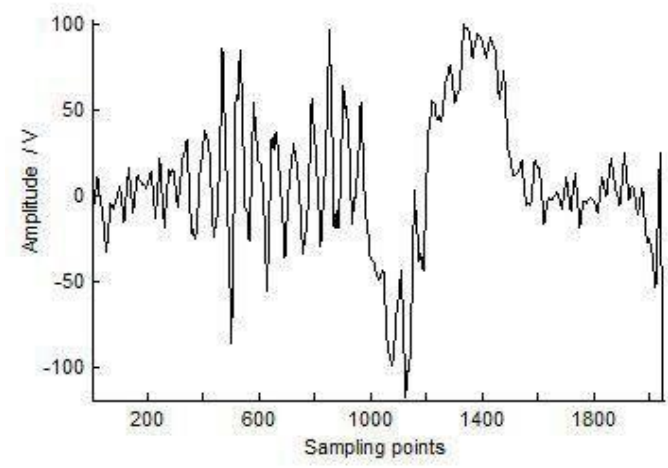

(c) Low-frequency approximation signal

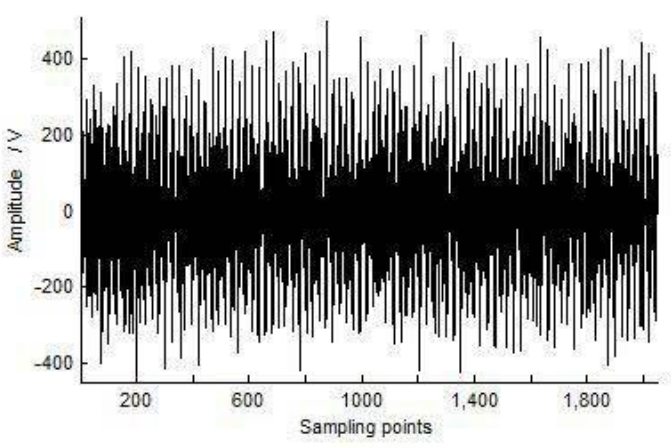

(d) High-frequency signal

Figure 2. The Effect of Wavelet Transform Denoising

Since there were some internal defects in the standing tree based on Figure 2(c), in order to confirm the existence of the defect, the stress wave tester ARBOTOM which was imported from Germany was used to detect the same location of the standing tree. Based on the stress wave software ZDPC-PROG, a two-dimensional cross-sectional map can be obtained which contains the stress wave propagation velocity, propagation paths, and wood decaying (Figure 3). The ID numbers in Figure 3 represent the locations of twelve sensors hung on the cross section of the standing tree. The stress wave two-dimensional cross-sectional map uses a "red - yellow - green" mode to indicate the propagation velocity. For example, the red area (darker) means that the stress wave propagation speed is relatively slow, indicating that this region is likely to have decay. The green area means that the stress wave propagation speed is relatively fast, indicating that there is no decay. The cross-sectional map can directly reflect the situation of growing internal decay through different color gradient (from green to yellow, from yellow to red). That is to say, the cross-sectional image is a visual representation of velocity changes, while the variation of velocity is the nature of the image color change [9]. Because there were only two ultrasonic probes being used to detect the standing tree, the ultrasonic signals were captured from the two test locations at ID_5291 and ID_5295. As can be seen from Fig. 3 , there was indeed some internal decay in the standing tree, which is consistent with the judgment of the ultrasonic signal.

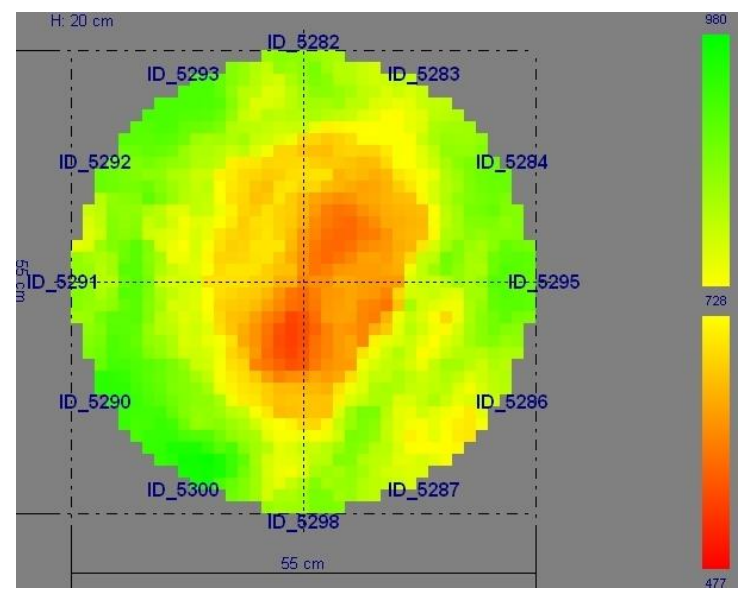

Figure 3. Cross-sectional Map Detected by Stress Wave 


\section{Conclusions and Discussion}

While detecting the defects in standing trees by using ultrasonic testing method, it is not very easy to visually identify the defects such as cracks, internal decay, and insect holes from the ultrasonic signals. If these defect characteristics in the ultrasonic signal can be detected, the diagnosis of internal defects in standing trees will be greatly enhanced, which has a significant practical value for identification of standing tree' quality. The ultrasonic testing signal is always mixed with some random noise and the presence of noise seriously interferes with the essential characteristics of the signal, which is not conducive to the signal analysis. Therefore, it is an urgent problem need to be resolved in testing the internal defects of standing trees that appropriate analytical methods should be used to extract the internal defect features from the original ultrasonic signal mixed with noise. The wavelet transform is a time-scale (time-frequency) analysis method for signal, which has the characteristics of multi-resolution analysis, but also has the ability to characterize the local signal characteristics in both the time and frequency domains, very suitable for the detection of transient anomalies carried by normal signals and showing their constituents. Therefore, wavelet transform was applied to perform denoising on the collected ultrasonic signal in the paper and the purpose was to make the ultrasonic signal after denoising not only to improve the SNR but also to project some detailed features for the signal.

The defect characteristics of ultrasonic signals are that when defects occur, the amplitude of the ultrasonic signal will change, while its phase and frequency are also changed. Wavelet decomposition is expanded on wavelet base, and breaks it down into different frequency bands. In fact, the wavelet decomposition of the ultrasonic signal is an infinite summation equation. Because of the sampling frequency and the limit of the computer, often this summation is intercepted to a desired scale and to get an approximate signal whose approximate extent is completely dependent on the choice of the wavelet base. In this study, we compared four wavelet base denoising parameters including Daubechies (db), Symlets (sym), Coiflets (coif) and Discrete Meyer (dmey), and $\mathrm{db} 3$ wavelet base had the best denoising effect. The variations of denoising parameters corresponding to the number of $\mathrm{db} 3$ wavelet decomposition levels (1- 8) were further analyzed and the decomposition level 4 was demonstrated the best. Meanwhile, the effects of wavelet denoising under different threshold states were compared and the hard rigrsure threshold was demonstrated the best. This is because heursure threshold is the combination sqtwolog threshold and rigsure threshold. When determining the threshold during the process of denoising, an appropriate threshold can be adaptively selected in sqtwolog threshold and rigsure threshold based on the size of the noise.

The singular points and irregular mutation parts in the ultrasonic testing signals often bring more important information, which are one of the important characteristics of the signals, especially in the detection of internal defects in the standing trees. Based on the reconstruction of the low and high frequency portions of the ultrasonic testing signal, the singularity of the ultrasonic testing signal can be checked. The reconstructed lowfrequency signal indicated that there were internal defects in the ultrasonic detection signal of the standing tree. Finally, in order to confirm the existence of the defect, the diagnosis result was compared with the stress wave testing result, which finally proved the judgment by using ultrasonic testing signal. Thus, the feasibility of applying wavelet transform in ultrasonic signal denoising for standing trees is proven. In addition, the results of this study can be combined with the variations of ultrasonic wave propagation parameters such as time, velocity, and modulus of elasticity to determine the internal defects, thereby more effectively improving the reliability and applicability of ultrasound technology in detecting the internal defects in the standing trees. 


\section{Acknowledgements}

The authors would like to acknowledge the support of the Fundamental Research Funds for the Central Universities (DL13BB19) and the Scientific Activities Support Program of Chinese State Forestry Administration for Returned Talents from Overseas (415003).

\section{References}

[1] X. Wang and S. J. Shen, "Advances in non-destructive testing for lumber", Journal of Beijing Forestry University, vol. 31, no. S1, (2009), pp. 202-205.

[2] L. H. Wang, X. C. Yang and K. H. Xu, "Current situations and research development of non-destructive testing for wood", Forest Engineering, vol. 17, no. 6, (2001), pp. 1-5.

[3] H. M. Yang and L. H. Wang, "Application of ultrasonic power spectrums in non-destructive testing hole defects in wood", Forest Engineering, vol. 21, no. 2, (2005), pp. 8-9.

[4] B. Yu, S. Gao, L. H. Wang, W. T. Xie and Y. T. Hou, "The study on the propagation law of ultrasonic wave in log", Forest Engineering, vol. 30, no. 1, (2014), pp. 92-96.

[5] S. Q. Song, H. D. Xu and L. H. Wang, "Ultrasonic wave propagation velocity in cross section of Simon Poplar standing trees", Journal of Northeast Forestry University, vol. 38, no. 5, (2010), pp. 40-44.

[6] H. Li and X. Y. Liu, "Ultrasonic non-destructive evaluation of the wood frame for the great bell", China Wood Industry, vol. 17, (2003), pp. 33-36.

[7] Z. X. Ge and W. Sha, "Wavelet analysis theory and realization in MATLABR2007", Publishing House of Electronics Industry, Beijing, (2007).

[8] C. Yang, D. M. Yu, Z. P. Xu and Y. Q. Wang, "Projected fringe patterns analysis based on wavelet transform", Journal of Tianjin University of Science \& Technology, vol. 22, no. 2, (2007), pp. 40-44.

[9] H. D. Xu, L. H. Wang and X. F. You, "Analysis of stress wave propagation in Hankow willow standing trees and stability assessment”, Scientia Silvae Sinicae, vol. 46, no. 8, (2010), pp. 145-150.

Authors

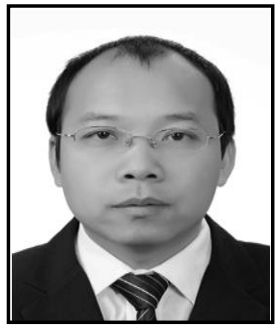

Wenshu Lin, He earned a B.E. degree and a M.S. degree in Forest Engineering from Northeast Forestry University, Harbin, P.R.China in 2003 and 2005, respectively. In 2011, he received a $\mathrm{Ph} . \mathrm{D}$. degree in Forest Resources Science from West Virginia University, USA. He is a Member of Forest Products Society and Society of Wood Science and Technology. Dr. Lin had several publications in esteemed journals such as Forest Products Journal, Wood and Fiber Science, and Computers and Electronics in Agriculture. Currently, he is an associate professor at Northeast Forestry University, Harbin, P. R. China. His major research interests include signal processing, nondestructive testing and evaluation of wood, and computer simulation and system modeling.

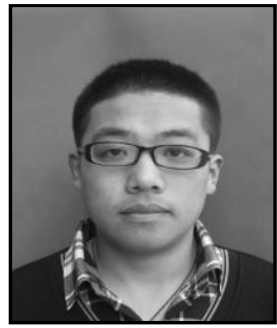

Haokai Xu, He earned a B.E. degree in Forest Engineering from Northeast Forestry University, Harbin, P.R.China in 2013. Currently, he is a master student in the Northeast Forestry University. His major research interests include signal processing, nondestructive testing and evaluation of wood, and computer simulation and system modeling. 


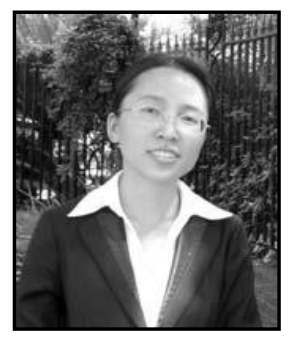

Jinzhuo Wu, He earned her B.E. degree and M.S. degree in Forest Engineering from Northeast Forestry University, Harbin, P. R. China in 2003 and 2005, respectively. In 2010, she obtained her $\mathrm{Ph} . \mathrm{D}$. degree in Forest Resources Science from West Virginia University, USA. She is a Member of Forest Products Society. Dr. $\mathrm{Wu}$ had several publications in esteemed journals such as Forest Products Journal, Wood and Fiber Science, Canadian Journal of Forest Research, and Journal of Agriculture and Resources Economics. 